\title{
Review of COVID-19 viral vector-based vaccines and COVID-19 variants
}

\author{
Rachana Vanaparthy ${ }^{1}$, Gisha Mohan², Deepa Vasireddy ${ }^{3}$, Paavani Atluri ${ }^{4}$ \\ ${ }^{1}$ Physicians for American Health Care Access, Philadelphia, PA, USA; \\ 2Department of Medicine, Suburban Community Hospital, East Norristown, Pennsylvania, USA; \\ ${ }^{3}$ Department of Pediatrics, Pediatric Group of Acadiana, Lafayette, Louisiana, USA; \\ ${ }^{4}$ Department of Medicine, Bay Area Hospital, Coos Bay, Oregon, USA
}

Article received 14 June, 2021; accepted 18 July, 2021

\section{SUMMARY}

The concept of viral vector-based vaccine was introduced in 1972 by Jackson et al and in 1982 Moss et al introduced the use of vaccinia virus as a transient gene expression vector. The technology has been used to make Ebola vaccines and now COVID-19 vaccines. There are two types of viral vector-based vaccines i.e. replicating and non-replicating. Non-replicating viral vector-based vaccines use replication-deficient viral vectors to deliver genetic material of a particular antigen to the host cell to induce immunity against the desired antigen. Replicating vector vaccines produce new viral particles in the cells they enter, which then go on to enter more new cells which will also make the vaccine antigen. Non-replicating vector-based vaccines are more commonly utilized. Adenovirus, vesicular stomatitis virus, vaccinia virus, adenovirus associated virus, retrovirus, lentivirus, cytomegalovirus, and sendai virus have been used as vectors. Current adenovi- rus vector-based vaccines being administered against SARS-CoV-2 infection are JNJ-78435735 by Johnson and Johnson (Janssen) along with Beth Israel Deaconess Medical Center, AZD1222 by Oxford-AstraZeneca, Sputnik V and Sputnik Light by Gamaleya Research Institute of Epidemiology and Microbiology, and Convidecia vaccine by CanSino Biologics. Of the five vaccines, the United States Food and Drug Administration (FDA) has approved Janssen vaccine for emergency use. Efficacy against COVID-19 variants has been found in all but the Convidecia vaccine so far. Heterologous prime-boost COVID-19 vaccination regimen may be the new face and more efficient immunization approach for enhanced immunity against COVID-19.

Keywords: COVID-19 vaccines, COVID-19 variants, Janssen, AstraZeneca, Sputnik V, Sputnik light, Convidecia.

\section{INTRODUCTION}

$\mathrm{T}_{\mathrm{in}}^{\mathrm{h}}$ he concept of viral vector-based vaccine was introduced in 1972 by Jackson et al and in 1982 Moss et al introduced the use of vaccinia virus as a transient gene expression vector [1-3]. As of May 2021, there has been a wide range of use of viral vectors as tools of gene therapy and vaccines. Two Ebola vaccines developed from viral vectors were used in Ebola outbreaks in West

$\overline{\text { Corresponding author }}$

Deepa Vasireddy

E-mail: dvasireddy@pgacadiana.com
Africa and in the Democratic Republic of Congo. The rVSV-ZEBOV vaccine is the first dose of Ebola vaccine approved in the United States to be used in December 2019 consisting of genetically engineered VSV virus. Zabdeno/Myabea Ebola vaccine is the second dose using modified vaccinia Ankara, a type of pox virus as the viral vector [4-6]. Genetically engineered adenovirus has been used to develop COVID-19 vaccines. It has shown to have increased efficacy by induction of immune responses and thereby increased cellular immunity. In this review article, we describe the different viral vector based COVID-19 vaccines, their mechanism of action, side effects, and efficacy against different variants. 


\section{Viral vector-based vaccines}

There are two types of viral vector-based vaccines i.e. replicating and non-replicating. Non-replicating viral vector-based vaccines use replication-deficient viral vectors to deliver genetic material of a particular antigen to the host cell to induce immunity against the desired antigen. As of June 2021, there are 7 viral vector-based vaccines in use. Of them, two are Ebola vaccines and five are COVID-19 vaccines [7]. Replicating vector vaccines produce new viral particles in the cells they enter, which then go on to enter more new cells which will also make the vaccine antigen.

Adenovirus, a double-stranded DNA virus discovered by Rowe and his colleagues in 1953 from human adenoid tissue, was effectively used for the development of these vaccines [8]. Adenovirus has a distinctive icosahedral structure with an outer protein encapsulation and compact double stranded DNA genome. It expresses 2 types of genes: early genes and late genes. Early genes are responsible for viral replication while late genes are responsible for virion release [9]. They are also used as vaccine vectors against numerous infections like HIV (Human immunodeficiency virus), malaria and also against a multitude of tumor associated antigens. They have the unique property to act as vaccine vectors and induce both innate and adaptive immune responses in mammalian hosts [10].
Other viruses such as vesicular stomatitis virus, vaccinia virus, adenovirus associated virus, retrovirus, lentivirus, cytomegalovirus, and sendai virus are used as vectors [11]. Various human clinical trials were conducted for viral vector vaccines against several infectious diseases including Zika virus, influenza viruses, respiratory syncytial virus, HIV, malaria before the vaccines targeting SARS COV-2 (Severe acute respiratory syndrome coronavirus 2) causing Covid [9].

\section{Mechanism of action of adenovirus as vaccine vector in COVID-19 vaccines}

The deletion of E1 genes terminates the viral replication. The compact genomic structure of adenovirus makes it a suitable option for the incorporation of a foreign DNA (deoxyribonucleic acid). Genes encoding the SARS-CoV-2 spike protein are isolated and introduced into the adenovirus vector after the gene sequencing. These viruses grow into specialized cells and further purification is done before developing into vaccines. The vaccine is then injected into the host which triggers both a humoral and cellular immune response and develops neutralizing antibodies. This results in the destruction of SARS-CoV-2 virus on future exposure with an actual SARS-Cov2 virus [12]. Current adenovirus vector-based vaccines against SARS-CoV-2 infection are JNJ-78435735 by Johnson and Johnson (Janssen) along with Beth Isra-

Table 1 - Viral vector-based COVID-19 vaccine characteristics.

\begin{tabular}{|c|c|c|c|}
\hline COVID-19 Vaccine & Dosage and administration & Storage & Variant efficacy \\
\hline $\begin{array}{l}\text { JANSSEN } \\
\text { (JNJ-78436735 } \\
\text { or Ad26.COV2.S) }\end{array}$ & $\begin{array}{l}\text { Single dose of } 0.5 \mathrm{ml} \\
\text { administered intramuscular } \\
\text { injection }\end{array}$ & $\begin{array}{l}\text { Up to three months at } 36-46^{\circ} \mathrm{F} \\
\left(2-8^{\circ} \mathrm{C}\right) \text { and up to } 2 \text { yrs at }-20^{\circ} \mathrm{C}\end{array}$ & $\begin{array}{l}\text { B.1.1.7 variant, B.1.351variant } \\
\text { and P.2 variant }\end{array}$ \\
\hline $\begin{array}{l}\text { ASTRAZENECA } \\
\text { (AZD-1222 or } \\
\text { ChAdOx1 nCoV-19) }\end{array}$ & $\begin{array}{l}\text { Two doses of vaccine } \\
\text { administered intramuscular } \\
\text { injection }\end{array}$ & $\begin{array}{l}\text { Can be stored and shipped } \\
\text { at }-20^{\circ} \mathrm{C}\end{array}$ & $\begin{array}{l}\text { B.1.1.7 variant, B.1.351 variant, } \\
\text { and effective in preventing } \\
\text { symptomatic COVID-19 infection } \\
\text { caused by non-B.1.1.7 strains }\end{array}$ \\
\hline $\begin{array}{l}\text { SPUTNIK V } \\
\text { (Gam-COVID-Vac) }\end{array}$ & $\begin{array}{l}\text { Two doses of vaccine } \\
\text { Intramuscular injection }\end{array}$ & $\begin{array}{l}\text { Two formulations } \\
\text { (frozen [Gam-COVID-Vac] } \\
\text { (storage temperature is }-18{ }^{\circ} \mathrm{C} \\
\left(0{ }^{\circ} \mathrm{F}\right) \text { ) and lyophilised [Gam- } \\
\text { COVID-Vac-Lyo] (storage } \\
\text { temperature is } 2-8^{\circ} \mathrm{C}\left(36-46{ }^{\circ} \mathrm{F}\right)\end{array}$ & $\begin{array}{l}\text { B.1.1.7 variant. However, there is } \\
\text { marked reduction in neutralizing } \\
\text { antibodies against B.1.351, P.1, } \\
\text { and B.1.1.28 variants }\end{array}$ \\
\hline SPUTNIK LIGHT & $\begin{array}{l}\text { Single dose of } 0.5 \mathrm{ml} \\
\text { intramuscular injection }\end{array}$ & $\begin{array}{l}\text { Stored at a normal refrigerator } \\
\text { temperature of } 2-8^{\circ} \mathrm{C}\end{array}$ & $\begin{array}{l}\text { As per Gamaleya center, } \\
\text { this vaccine is effective against } \\
\text { all new variants }\end{array}$ \\
\hline $\begin{array}{l}\text { CONVIDECIA } \\
(\text { Ad5-nCoV) }\end{array}$ & $\begin{array}{l}\text { Single dose of } 0.5 \mathrm{ml} \\
\text { intramuscular injection }\end{array}$ & $\begin{array}{l}\text { Stored at a normal refrigerator } \\
\text { temperature of } 2-8^{\circ} \mathrm{C}\end{array}$ & $\begin{array}{l}\text { No clear data yet on variant } \\
\text { efficacy }\end{array}$ \\
\hline
\end{tabular}


el Deaconess Medical Center, AZD1222 by Oxford-AstraZeneca, Sputnik V and Sputnik Light by Gamaleya Research Institute of epidemiology and microbiology, and CanSino vaccine by CanSino Inc. Of the five vaccines, the United States Food and Drug Administration (FDA) has approved Janssen vaccine for emergency use [13].

Janssen utilized the gene encoding the coronavirus spike protein to replicate the replication-deficient adenovirus 26 . The genetically engineered adenovirus 26 is developed into a vaccine, the vaccine once injected into the host cell is engulfed by the cell [14]. The AstraZeneca vaccine is a chimpanzee adenovirus vaccine vector made up of replication deficient chimpanzee adenoviral vector chA$\mathrm{dOx} 1$. It has been genetically engineered to match the SARS-CoV-2 spike protein and is incorporated with the surface spike protein genetic sequence of this surface spike protein [15]. Sputnik V utilizes replication-deficient adenovirus as a vector to deliver the vaccine. The unique feature is the use of 2 types of adenovirus as the vector Ad26 and Ad5 whereas Sputnik light vaccine utilizes the first component of Sputnik V, recombinant human adenovirus serotype number 26 (rAd26). They are genetically engineered by inserting a gene encoding spike(S) protein of SARS-CoV-2. Once inside the cell's nucleus the spike protein gene is copied into messenger RNA (mRNA) [16]. The mRNA leaves the nucleus and starts building spike proteins, resulting in activation of the immune response. Humoral and cell-mediated immune responses are activated after the first dose. Memory B Cells and memory T-cells result in long term immune response which later fights against the virus on exposure.

\section{JANSSEN (JNJ-78436735 or AD26.COV2.S)}

JNJ-78436735 or Ad26.COV2.S is an adenoviral vector-based vaccine against SARS-CoV-2 developed by Janssen pharmaceuticals of Johnson and Johnson in collaboration with Beth Israel Deaconess Medical Center. The Janssen vaccine was approved for emergency use by United States food and drug administration (USFDA) on 28th February 2021 and its use had been suspended on April 13th 2021 because of the 6 reported cases of cerebral venous thrombosis and thrombocytopenia. The suspension was lifted and vaccine use was resumed on 23rd April 2021 by USFDA. It was granted authorization by European medicine agencies.

The unique characteristic of Janssen is it requires only a single dose of $0.5 \mathrm{~mL}$ administered IM and can be refrigerated for up to three months at 36$46^{\circ} \mathrm{F}\left(2-8^{\circ} \mathrm{C}\right)$ and up to 2 yrs at $-20^{\circ} \mathrm{C}[17,18]$.

\section{Vaccine dose and interval}

The Janssen vaccine is a single dose with intramuscular administration. The vaccine should be administered with a minimum interval of 14 days before or after administration of any other vaccine.

\section{Efficacy on variants}

The ENSEMBLE is an international, randomized, double-blind, placebo-controlled, phase 3, pivotal trial aimed to determine the safety, efficacy and immunogenicity of a single dose of vaccine in adults. The data from ENSEMBLE study after 28 days of vaccination has proven $66 \%$ of efficacy against moderate to severe or critical COVID-19 across all countries, $72 \%$ of efficacy against moderate to severe or critical COVID-19 in the US and $85 \%$ of efficacy against severe COVID-19 globally, including the US, and complete protection against COVID-19 related hospitalizations, and no COVID-19 related deaths were reported in the vaccine group. The clinical trial was conducted in 17 countries including the United States, South Africa, Mexico, Peru, Argentina, Chile, etc with a high incidence of COVID-19 infection and where variants were emerging. Janssen's vaccine efficacy was $75 \%$ against the B.1.1.7 variant (known as alpha variant or UK variant) and $57 \%$ against B.1.351 (known as beta variant or South African variant). However, in severe/critical patients the vaccine efficacy was $86 \%$ in US (\% variant: 96\% D614G and 3\% CAL.20C), 88\% in Brazil (\% variant: $69 \%$ P.2 lineage also known as zeta variant or Brazil variant and 31\% D614G), and 82\% in South Africa (\% variant: 95\% B.1.351 and 3\% D614G [17].

\section{Countries' approval}

As of June 4th, 2021, 42 countries approved the Janssen vaccine including Austria, Bahrain, Belgium, Brazil, Bulgaria, Canada, Colombia, Croatia, Cyprus, Czechia, Denmark, Estonia, Finland, France, Germany, Greece, Hungary, Iceland, Ireland, Italy, Latvia, Liechtenstein, Lithuania, 
Luxembourg, Malta, Netherlands, Norway, Philippines, Poland, Portugal, Republic of Korea, Romania, Slovakia, Slovenia, South Africa, Spain, Sweden, Switzerland, Thailand, Tunisia, United States of America, and Zambia [18].

\section{Side effect profile}

According to the data from the ENSEMBLE study and the Summary of Product Characteristics of Janssen's vaccine, the common side effects are injection site pain, erythema and swelling. Other systemic side effects are headache, fatigue, myalgia, nausea, fever and contraindication in patients with severe anaphylactic reactions. These side effects were mostly seen within the 7 days of vaccination. Interestingly, these are more common in individuals aged 18-59 years than that of individuals aged 60 years and older $[19,20]$. Rare side effects including convulsions, tinnitus, peripheral neuropathy, Guillain-Barrè syndrome, and Bell's palsy were reported in a few patients with predisposing medical conditions [17].

The Janssen vaccine received approval for emergency use by the United States Food and Drug Administration on February 27, 2021 for individuals of 18 years and above [21]. Since then, nearly 8,057,660 people have received the Janssen vaccine. On April 13, 2021, the FDA and Centers for Disease Control and Prevention (CDC) reported a total of 15 cases of rare and severe types of blood clot combined with low platelet levels, known as Thrombosis with thrombocytopenia syndrome (TTS) or Vaccine-induced thrombotic thrombocytopenia. This resulted in a temporary pause of vaccine administration [22]. A patient is considered to have TTS when they meet the following criteria: COVID-19 vaccine (Janssen or AstraZeneca) administration, venous or arterial thrombosis (commonly cerebral or abdominal), thrombocytopenia $(\leq 150,000)$ and positive PF4 'HIT' (heparin-induced thrombocytopenia) ELISA [23]. Brighton Collaboration has updated the definition to include arterial and vein thrombosis in various locations beyond cerebral like portal, hepatic, mesenteric, splenic, pulmonary, brachial, lower extremities, and carotid thrombosis [24]. In general, d-dimer levels are elevated in elderly hospitalized patients with COVID-19 infection requiring anticoagulation. Hence, there is increased risk of ischemic stroke [25]. A study by Baldini et al and Mowla et al mentions that the incidence of CVST is low but there is a relative risk which is potentially comorbid. But according to Taquet et al, who reviewed 537,913 COVID-19 cases has concluded that the risk of CVST and portal vein thrombosis (PVT) is significantly increased after COVID-19 infection. Till date, there is no data about the pathophysiology and potential prevention of CVST in COVID-19 infection [26-28].

In a report published by CDC, the median age of the 15 patients was 37 years (range 18-59 years), median time to symptom onset was 8 days (range 6-15 days) and all cases were females. Other common risk factors among these patients were oral contraceptive use, obesity, hypertension, and hypothyroidism. Among the 15 patients, 12 patients have reported cerebral venous sinus thrombosis (CVST), others reported with portal vein thrombosis (PVT), lower extremity vein thrombosis, and pulmonary artery thrombosis, 2 patients had previous history of COVID-19 infection. The common sites of CVST are transverse, sigmoid, straight, superior sagittal, inferior sagittal, cortical veins and confluence of sinuses. Most of the patients with CSVT reported intracerebral hemorrhage. The initial presentation with CSVT was associated with headaches mostly after 6 days of vaccination, fever with chills, malaise, lethargy, nausea, vomiting, and abdominal pain. These were followed by severe headache with neck stiffness or pain, unilateral weakness, speech difficulty, gaze deviation, loss of consciousness, and seizure. These patients received non-heparin anticoagulants, platelet transfusion, and intravenous immunoglobulin. Heparin was given only to patients with negative PF4 'HIT' antibodies. As of April 21, 2021, 5 patients were discharged home, 7 patients were still hospitalized including the intensive care unit (ICU), and 3 patients experienced death [29].

Given the potential benefits outweigh the risks, the US FDA has lifted the pause on April 24, 2021 with the intention to vaccinate a large population and control the spread of COVID-19 infection [30].

\section{ASTRAZENECA}

\section{(AZD-1222 or ChAdOx1 nCoV-19)}

AZD1222 vaccine formerly called ChAdOx $1 \mathrm{nCoV}-$ 19 also known by other names as Oxford-AstraZeneca Vaccine, Covishield, and Vaxzevria. AZD1222 was developed by Oxford University, 
Jenner institute and AstraZeneca. It is co-invented by the University of Oxford and its spin-out company, Vaccitech. This vaccine is also manufactured by the Serum Institute of India with the name Covishield. The vaccine can be stored at $2-8{ }^{\circ} \mathrm{C}(36-46$ $\left.{ }^{\circ} \mathrm{F}\right)$ for 6 months [31].

Vaccine dose and interval

Two doses of vaccine administered IM with a dosing interval of 12 weeks or more [32].

\section{Efficacy on variants}

From the phase 1 and 2 clinical trial data, the AstraZeneca vaccine had shown significant efficacy of $64.1 \%$ after at least a single dose against symptomatic disease and $70.4 \%$ after two doses, with no safety concerns. The phase 3 study showed a vaccine efficacy of $76 \%$ at preventing symptomatic COVID-19 infection and 100\% efficacy at preventing severe or critical disease and hospitalizations. The efficacy and immunogenicity of this vaccine have not been assessed and estimated to be lower in immunocompromised individuals including those receiving immunosuppressive therapy [28, 33, 34]. The two doses of the AstraZeneca vaccine have shown $61.7 \%$ effectiveness against the B.1.1.7 (UK) variant, 10.4\% against mild to moderate due to B.1.351 (South Africa) variant, and $77.3 \%$ against other variants. This vaccine is $81.5 \%$ effective in preventing symptomatic COVID-19 infection caused by non-B.1.1.7 strains $[35,36]$.

\section{Countries' approval}

As of June 4th, 2021, 99 countries approved Vaxzevria vaccine including Albania, Angola, Argentina, Australia, Austria, Belgium, Belize, Bosnia and Herzegovina, Botswana, Brazil, Brunei Darussalam, Bulgaria, Cambodia, Canada, Chile, Colombia, Costa Rica, Croatia, Cyprus, Czechia, Côte d'Ivoire, Democratic Republic of the Congo, Denmark, Dominican Republic, Ecuador, Egypt, El Salvador, Estonia, Eswatini, Fiji, Finland, France, Gambia, Georgia, Germany, Ghana, Greece, Grenada, Guatemala, Guyana, Hungary, Iceland, India, Indonesia, Iran (Islamic Republic of), Iraq, Ireland, Italy, Jamaica, Japan, Jordan, Kenya, Kosovo, Kuwait, Latvia, Liechtenstein, Lithuania, Luxembourg, Malawi, Malaysia, Mali, Malta, Mauritius, Mexico, Mongolia, Morocco, Netherlands, Nigeria, Oman, Pakistan, Papua
New Guinea, Philippines, Poland, Portugal, Republic of Korea, Republic of Moldova, Romania, Rwanda, Sao Tome and Principe, Saudi Arabia, Senegal, Serbia, Slovakia, Slovenia, Spain, Sudan, Sweden, Taiwan, Thailand, Timor-Leste, Uganda, United Kingdom of Great Britain and Northern Ireland, Uzbekistan, Viet Nam, Yemen, and Zambia [37].

\section{Side effect profile}

Similar to other vaccines, the most common adverse effects are local injection site pain, tenderness, erythema, and swelling, nausea and vomiting, fever with chills, muscle ache, headache, and malaise which are predominantly seen on the day 1 after vaccination. A rare complication of neutropenia, hemolytic anemia, and transverse myelitis was seen $[28,38,39]$. The vaccine is contraindicated in patients with hypersensitivity and anaphylactic reactions, anxiety, acute infection or severe febrile illness, and with history of thrombosis or thrombocytopenia [40].

By March 10, 2021, 5 million people had received this vaccine in Europe. Among them, 30 cases of thromboembolic events were reported. This resulted in a temporary 2 week pause in many countries including Denmark, Thailand, Norway, Iceland, Bulgaria, Luxembourg, Estonia, Lithuania, Latvia, Austria, and Italy [41]. On 22 March, 2021, the European Medicines Agency's (EMA) safety committee noted the blood clots in the veins of the brain, abdomen, and the arteries in patients with low levels of platelets. The committee conducted an in-depth review of 62 cases of CSVT and 24 cases of splanchnic vein thrombosis. This is estimated to be 1 in 100,000 vaccinated people. Of these, 18 cases were fatal. Similar to the Janssen vaccine, most of the reported cases were females under the age of 60 years and occurred within 2 weeks of their first dose [42].

As mentioned above, the pathogenesis for the formation of blood clots is not clear but the possible mechanism for this condition is thought to be that the vaccine can trigger an immune response similar to that of heparin-induced thrombocytopenia in patients treated with heparin. The patients can present with shortness of breath, chest pain, pedal edema, persistent abdominal pain, other neurological symptoms like severe and persistent headaches or blurred vision, and petechiae beyond the injection site [40, 42]. 


\section{SPUTNIK V (Gam-COVID-Vac)}

Gam-COVID-Vac trade-named Sputnik V, so named after the first artificial satellite. It is an adenoviral vector-based COVID19 vaccine developed by the Gamaleya Research Institute of epidemiology and microbiology. The vaccine was designed with two recombinant adenovirus vectors and was developed as two formulations (frozen [Gam-COVID-Vac] (storage temperature -18 $\left.{ }^{\circ} \mathrm{C}\left(0{ }^{\circ} \mathrm{F}\right)\right)$ and lyophilised [Gam-COVID-Vac-Lyo] (storage temperature $2-8{ }^{\circ} \mathrm{C}\left(36-46^{\circ} \mathrm{F}\right)$.

\section{Vaccine dose and interval}

Two doses of vaccine administered IM with a dosing interval of 3 weeks which may increase up to 3 months [43].

\section{Efficacy on variants}

The phase 1 and 2 clinical trials showed a good safety profile and also induced strong humoral and cellular immune response against SARS$\mathrm{CoV}-2$. The phase 3 data of 20,000 participants has demonstrated $91.6 \%$ effectiveness 21 days after the first dose of vaccine and after the day of receiving the second dose; in the recent press release by Gamaleya center on 19 April, 2021, according to the data from 38 million vaccinated individuals this vaccine has demonstrated $97.6 \%$ efficacy against SARS-CoV-19 making it the most effective vaccine in the world, and 100\% effective against severe COVID-19 infection [44, 45]. The Sputnik V vaccine is effective against the B.1.1.7 variant. However, there is marked reduction in neutralizing antibodies against B.1.351, P.1, and B.1.1.28 variants [46].

\section{Countries' approval}

As of June 4th, 2021, the Sputnik V vaccine is approved in 68 countries including Albania, Algeria, Angola, Antigua and Barbuda, Argentina, Armenia, Azerbaijan, Bahrain, Bangladesh, Belarus, Bolivia (Plurinational State of), Bosnia and Herzegovina, Cameroon, Djibouti, Egypt, Gabon, Ghana, Guatemala, Guinea, Guyana, Honduras, Hungary, India, Iran (Islamic Republic of), Iraq, Jordan, Kazakhstan, Kenya, Kyrgyzstan, Lao People's Democratic Republic, Lebanon, Libya, Mali, Mauritius, Mexico, Mongolia, Montenegro, Morocco, Myanmar, Namibia, Nepal, Nicaragua, North Macedonia, Pakistan, Panama, Paraguay,
Philippines, Republic of Moldova, Republic of the Congo, Russian Federation, Saint Vincent and the Grenadines, San Marino, Serbia, Seychelles, Slovakia, Sri Lanka, Syrian Arab Republic, Tunisia, Turkmenistan, United Arab Emirates, Uzbekistan, Venezuela (Bolivarian Republic of), Viet Nam, West Bank, and Zimbabwe [47].

\section{Side effect profile}

The phase 1 and 2 clinical trial data have reported the most common systemic and local reactions. Local reactions were injection site pain and erythema. Hyperthermia, chills, headache, asthenia, muscle ache, and joint pains were reported, as well [48]. Despite all vaccines described here being developed on adenoviral vector-based platforms, each vaccine has significant differences in their structure and production. AstraZeneca used chimpanzee adenovirus while Janssen used human adenovirus serotype to deliver antigens. However, Sputnik V used adenovirus serotypes 5 and 26. Hence, the safety data is not comparable to one another. A comprehensive analysis of adverse events during clinical trials and over the course of mass vaccinations with the Sputnik $V$ vaccine published by Gamaleya center on April 14, 2021, has shown that there were no reported cases of cerebral venous sinus thrombosis [49].

\section{SPUTNIK LIGHT}

Sputnik light vaccine is a single dose vaccine based on the Ad26 vector developed by Gamaleya research institute of epidemiology and microbiology. It can be stored at a normal refrigerator temperature of 2-8 degree Celsius. It has an effectiveness of $79.4 \%$ suited for acute outbreaks [16].

\section{Vaccine dose and interval}

It's administered as a single dose of $0.5 \mathrm{~mL}$ intramuscular injection.

\section{Efficacy on variants}

Similar to the Sputnik V vaccine, Sputnik light, a single dose vaccine, is developed with an adenovirus vector-based platform. This vaccine utilized the first component of Sputnik V, recombinant human adenovirus serotype number 26 (rAd26). The phase 1 and 2 clinical trial data demonstrated an efficacy of $79.4 \% 28$ days after the vaccination in Russia's mass vaccination program during 5 
December 2020 to 15 April 2021. As per the Gamaleya center, this vaccine is effective against all new variants [50].

\section{Countries approved}

As of June 4th, 2021, Russia and Angola authorized the Sputnik light vaccine [50, 51].

\section{Side effect profile}

As such, no serious adverse events including cerebral venous thrombosis have been reported [50].

\section{CONVIDECIA (Ad5-nCoV)}

Convidecia is a viral vector-based vaccine similar to AZD1222 and Sputnik V. It's developed by CanSino Biologics. This vaccine can be stored in a normal refrigerator at temperatures of 2-8 degree celsius [52].

Vaccine dose and interval

It's administered IM and a single dose of $0.5 \mathrm{~mL}$ is given.

\section{Efficacy on variants}

Data from the late-stage clinical trials, single-dose vaccine, CanSino, is $65.7 \%$ effective in preventing symptomatic and $90.98 \%$ effective in preventing severe COVID-19 infection [53]. But the efficacy dropped from $68.83 \%$ after two weeks to $65.28 \%$ after four weeks. Hence, a booster shot may be required to develop desired neutralizing antibodies and the efficacy might also increase to 90\% [54]. There is no clear data on the efficacy of the CanSino vaccine on the variants.

\section{Countries approved}

As of June 4th, 2021, the CanSino vaccine is approved in 5 countries including Chile, China, Hungary, Mexico, and Pakistan [55].

\section{Side effect profile}

The side effects reported in the clinical trials were mild/moderate and no serious events within 28 days of vaccination. The common adverse events reported in the clinical trials were high fever $(42 \%)$, fatigue $(44 \%)$, headache $(39 \%)$, muscle ache $(17 \%)$, joint pains, and injection site pain (54\%). All these events lasted no more than 48 hours [56]. Seven days after vaccination, a few patients reported an increase in bilirubin $(8 \%)$, alanine aminotransferase (9\%), and fasting blood sugars (4\%) [57].

\section{COVID-19 VARIANTS AND VACCINATION EFFORTS}

As of July, 2021, the Center for Disease Control and Prevention (CDC) has classified variants into Variant of Interest, Variant of Concern, and Variant of High Consequence. Currently, B.1.1.7 (alpha), B.1.351 (beta), B.1.617.2 (delta), P.1 (gamma) are classified under variants of concern. B.1.427 (epsilon), B.1.429 (epsilon), B.1.525 (eta), B.1.526 (lota), B.1.617.1 (kappa), B.1.617.3, P.2 (zeta) are classified under variants of interest $[58,59]$. The new variant, delta plus, has the additional spike mutation of the widespread delta variant which was initially identified in India and is now raising concerns all over the world [60]. As of July 8th, 2021 , about $24.9 \%$ of the world's population has been vaccinated with at least one dose of a COVID-19 vaccine [61]. In the USA, $47.5 \%$ of the total population had been fully vaccinated by the end of June 2021. The population over age 65 years was the most vaccinated age group in the USA [62]. At the time of this paper, China, USA and India were the countries with the most fully vaccinated people [61].

\section{HETEROLOGOUS PRIME-BOOST COVID-19 VACCINATION}

In February, a study called 'Com-Cov' initiated by the UK's National Institute for Health Research, enrolled 800 volunteers to study the COVID-19 Heterologous Prime Boost against COVID-19 virus. This study is tested in different combinations. So far, patients were divided into eight different arms, including AstraZeneca and AstraZeneca 28 days apart, AstraZeneca and AstraZeneca 12 weeks apart, Pfizer and Pfizer 28 days apart, Pfizer and Pfizer 12 weeks apart, AstraZeneca and Pfizer 28 days apart, AstraZeneca and Pfizer 12 weeks apart, Pfizer and AstraZeneca 28 days apart, Pfizer and AstraZeneca 12 weeks apart as first and second doses respectively. This study will run for 13 months and data is analyzed [63]. In April, the Carlos III Health Institute in Madrid conducted the Spanish CombivacS trial with 663 participants who received the first dose of the AstraZeneca vaccine. Of them, 441 patients received 
Pfizer vaccine as their second dose after 8 weeks after their first dose and 232 patients did not receive any booster. This study published on May 19th, 2021 revealed that the patients who received the Pfizer vaccine as their second had developed higher levels of neutralizing antibodies than those receiving two doses of AstraZeneca vaccine. This implies that the combination of both AstraZeneca and Pfizer-BioNTech vaccines have boosted immunity by improving Th1-biased T cell responses against SARS-CoV-2 virus. These studies also noticed the milder side effects in patients who received the combination vaccine $[64,65]$.

\section{CONCLUSIONS}

Through our review article, we discussed a brief overview of the five vector-based vaccines for COVID-19, mechanism of action, clinically serious and potentially life-threatening adverse events caused with Janssen and AstraZeneca vaccines. Though the incidence of TTS is low, it is very important to monitor the signs and symptoms including shortness of breath, chest pain, leg swelling, pain abdomen, neurological symptoms, petechiae for early identification and prevent complications. At the time of this paper, there were 16 non-replicating viral vector-based vaccines and 2 replicating viral vector-based vaccines in clinical development phase. The routes of administration being considered for the vaccines in the clinical development phase were intramuscular, intranasal, oral and subcutaneous. There are 21 non-replicating viral vector-based vaccines in pre clinical developmental stage [66]. Heterologous prime and boost concept of vaccination may be the new face of COVID-19 vaccination for more effective results.

\section{Funding}

No funding or grants were required for this study.

\section{Conflict of interest}

All authors declare that there exist no commercial or financial relationships that could, in any way, lead to a potential conflict of interest.

\section{REFERENCES}

[1] Jackson DA, Symons RH, Berg P. Biochemical method for inserting new genetic information into DNA of simian virus 40: circular SV40 DNA molecules containing lambda phage genes and the galactose operon of Escherichia coli. 1972. Biotechnology. 1992; 24, 11-16.

[2] Mackett M, Smith GL, Moss B. Vaccinia virus: a selectable eukaryotic cloning and expression vector. Proc Natl Acad Sci USA. 1982; 79 (23), 7415-9.

[3] Panicali D, Paoletti E. Construction of poxviruses as cloning vectors: insertion of the thymidine kinase gene from herpes simplex virus into the DNA of infectious vaccinia virus. Proc Natl Acad Sci USA. 1982; 79 (16), 4927-31.

[4] Understanding Viral Vector COVID-19 Vaccines. Centers for Disease Control and Prevention. https:// www.cdc.gov / coronavirus / 2019-ncov / vaccines / different-vaccines / viralvector.html\#: : text=Viral $\% 20$ vector $\% 20$ vaccines $\% 20$ use $\% 20$ a,getting $\% 20$ sick $\% 20$ with\%20COVID\%2D19. Accessed June 9, 2021.

[5] Marzi A, Ebihara H, Callison J, et al. Vesicular stomatitis virus-based Ebola vaccines with improved cross-protective efficacy. J Infect Dis. 2011; 204 (Suppl 3), S1066-74.

[6] A Safety and Immunogenicity Study of Heterologous Prime-Boost Ebola Vaccine Regimens in Healthy Participants - Full Text View. Full Text View - ClinicalTrials.gov. https:/ / clinicaltrials.gov/ct2/show/NCT02313077. Accessed June 9, 2021.

[7] Ura T, Okuda K, Shimada M. Developments in Viral Vector-Based Vaccines. Vaccines (Basel). 2014; 2 (3), 624-41.

[8] Rowe, W.P. et al. Isolation of a cytopathogenic agent from human adenoids undergoing spontaneous degeneration in tissue culture. Proc Soc Exp Biol Med. 1953; 84, 570-3.

[9] Understanding and Explaining Viral Vector COVID-19 Vaccines". U.S. Centers for Disease Control and Prevention. 2021-02-25. Retrieved 2021-04-02.

[10] Ghebremedhin, B. Human adenovirus: viral pathogen with increasing importance. Eur J Microbiol Immunol. 2014; 4 (1), 2633.

[11] Tatsis N, Ertl HC. Adenoviruses as vaccine vectors. Mol Ther. 2004; 10 (4), 616-29.

[12] Feng L, Wang Q, Shan C, et al. An adenovirus-vectored COVID-19 vaccine confers protection from SARSCOV-2 challenge in rhesus macaques. Nat Commun. 2020; 11 (1), 4207.

[13] Vasireddy D., Atluri P, Malayala S, Vanaparthy R, and Mohan G. Review of COVID-19 vaccines approved in the United States of America for emergency use. J Clin Med Res. 2021; 3 (4), 204-13.

[14] Corum J, Zimmer C. How the Johnson \& Johnson Vaccine Works. The New York Times. https://www. nytimes.com/interactive/2020/health/johnson-johnson-covid-19-vaccine.html. Published December 18, 2020. Accessed June 9, 2021.

[15] Wise J. COVID-19: New data on Oxford AstraZeneca vaccine backs 12 week dosing interval. The BMJ. 
https://doi.org/10.1136/bmj.n326. Published February 3, 2021. Accessed June 9, 2021.

[16] Meredith S. Russia authorizes use of "Sputnik Light," a one-shot Covid vaccine it says is 79\% effective. CNBC. Published May 6, 2021. Accessed July 29, 2021. https: / / www.cnbc.com/2021/05/06/sputnik-lightrussia-authorizes-use-of-one-shot-covid-vaccine.html

[17] Overview of Janssen's Single-Dose COVID-19 Vaccine, Ad26.COV2.S Janssen Pharmaceutical Companies of Johnson \& Johnson. US Centers for Disease Control and Prevention Advisory Committee on Immunization Practices. CDC.gov. Published February 28, 2021. Accessed June 6, 2021.

[18] Janssen (Johnson \&amp; Johnson): Ad26.COV2.S. COVID19 Vaccine Tracker. https://covid19.trackvaccines.org/vaccines/1/. Accessed June 6, 2021.

[19] Johnson \& Johnson Single-Shot COVID-19 Vaccine Phase 3 Data Published in New England Journal of Medicine. Janssen. https://www.janssen.com/ johnson-johnson-single-shot-covid-19-vaccine-phase3-data-published-new-england-journal-medicine. Published April 21, 2021. Accessed June 6, 2021.

[20] Janssen COVID-19 Emergency Use Authorization (EUA) Official Website. Janssen COVID-19 Vaccine Official Website. https://www.janssencovid19vaccine. com/. Accessed June 6, 2021.

[21] Johnson \& Johnson COVID-19 Vaccine Authorized by U.S. FDA For Emergency Use. Janssen. https:/ / www.janssen.com/johnson-johnson-covid-19-vaccineauthorized-us-fda-emergency-use. Published February 27, 2021. Accessed June 6, 2021.

[22] FDA and CDC Lift Recommended Pause on Johnson \& Johnson (Janssen) COVID-19 Vaccine Use Following Thorough Safety Review. U.S. Food and Drug Administration. Accessed June 6, 2021.

https://www.fda.gov/news-events/press-announcements/fda-and-cdc-lift-recommended-pause-johnsonjohnson-janssen-covid-19-vaccine-use-following-thorough.

[23] von Hundelshausen P, Lorenz R, Siess W, Weber C. Vaccine-Induced Immune Thrombotic Thrombocytopenia (VITT): targeting pathomechanisms with bruton tyrosine kinase inhibitors. Thromb Haemost. 2021; 10.1055/a-1481-3039; doi:10.1055/a-1481-3039.

[24] Interim Case Definition of Thrombosis with Thrombocytopenia Syndrome (TTS). Brighton Collaboration. https://brightoncollaboration.us/thrombosis-withthrombocytopenia-syndrome-interim-case-definition/. Published May 24, 2021. Accessed June 6, 2021.

[25] Katsanos AH, Palaiodimou L, Zand R, et al. The Impact of SARS-CoV-2 on Stroke Epidemiology and Care: A Meta-Analysis. COVID. https://covid19.elsevierpure.com/de/publications/the-impact-of-sars-cov-2on-stroke-epidemiology-and-care-a-meta-a. Published December 18, 2020. Accessed June 6, 2021.

[26] Baldini T, Asioli GM, Romoli M, et al. Cerebral venous thrombosis and severe acute respiratory syndrome coronavirus-2 infection: A systematic review and meta-analysis. Eur J Neurol. 2021; 10.1111/ene.14727. doi:10.1111/ene.14727.

[27] Ostovan VR, Foroughi R, Rostami M, et al. Cerebral venous sinus thrombosis associated with COVID-19: a case series and literature. J Neurol. 2021; 1-12. doi:10.1007/s00415-021-10450-8.

[28] Alexander JL, Powell N, British Society of Gastroenterology Inflammatory Bowel Disease section and IBD Clinical Research Group. SARS-CoV-2 vaccination in immunosuppressed patients with inflammatory bowel disease: should our approach change? Lancet Gastroenterol Hepatol. 2021;6(7):528-529.Alexander JL, Powell $\mathrm{N}$ on behalf of the British Society of Gastroenterology Inflammatory Bowel Disease section and IBD Clinical Research Group. SARS-CoV-2 vaccination in immunosuppressed patients with inflammatory bowel disease: should our approach change? Lancet Gastroenterol Hepatol. 2021; 6 (7), 528-529.

[29] Shimabukuro T. Thrombosis with thrombocytopenia syndrome (TTS) following Janssen COVID-19 vaccine. National Center for Immunization \& Respiratory Diseases. www.cdc.gov/vaccines/acip. Published April 23, 2021. Accessed June 7, 2021.

[30] NCDHHS. https://www.ncdhhs.gov/news/pressreleases/cdc-and-fda-lift-recommended-pause-johnson-johnson-covid-19-vaccine-following safety review. Accessed June 7, 2021.

[31] NHS Choices. https://www.sps.nhs.uk/articles/ storage-requirements-for-each-covid-19-vaccine/. Accessed June 9, 2021.

[32] Wise J. COVID-19: New data on Oxford AstraZeneca vaccine backs 12 week dosing interval. BMJ. 2021; 372:n326. Published 2021 Feb 3. doi:10.1136/bmj.n326.

[33] Voysey M, Clemens SAC, Madhi SA, et al. Safety and efficacy of the ChAdOx1 nCoV-19 vaccine (AZD1222) against SARS-CoV-2: an interim analysis of four randomised controlled trials in Brazil, South Africa, and the UK. Lancet. 2021; 397(10269), 99-111.- need to add this as reference for lines 223-225.

[34] AZD1222 US Phase III primary analysis confirms safety and efficacy. AstraZeneca. https://www.astrazeneca.com/media-centre/press-releases / 2021/ azd1222-us-phase-iii-primary-analysis-confirms-safety-and-efficacy.html. Published March 25, 2021. Accessed June 7, 2021

[35] Madhi SA, Baillie V, Cutland CL, et al. Efficacy of the ChAdOx1 nCoV-19 COVID-19 vaccine against the B.1.351 variant. N Engl J Med. 2021; 384 (20), 1885-8.

[36] Lianna Matt McLernon | News Writer | CIDRAP News | Mar 31 2021. AstraZeneca COVID vaccine 70\% effective vs B117 variant. CIDRAP. https://www.cidrap.umn.edu/news-perspective/2021/03/astrazeneca-covid-vaccine-70-effective-vs-b117-variant. Published March 31, 2021. Accessed June 7, 2021. 
[37] Oxford/AstraZeneca: AZD1222. COVID19 Vaccine Tracker. https://covid19.trackvaccines.org/vaccines/4/. Accessed June 7, 2021.

[38] Folegatti PM, Ewer KJ, Aley PK, et al. Safety and immunogenicity of the ChAdOx1 nCoV-19 vaccine against SARS-CoV-2: a preliminary report of a phase $1 / 2$, single-blind, randomised controlled trial Lancet. 2020; 396 (10249), 467-78.

[39] UK and EU regulatory agencies confirm COVID-19 Vaccine AstraZeneca is safe and effective. AstraZeneca. https://www.astrazeneca.com/content/astraz/media-centre/press-releases/2021/uk-and-eu-regulatoryagencies-confirm-covid-19-vaccine-astrazeneca-is-safeand-effective.html. Published March 18, 2021. Accessed June 9, 2021.

[40] Wise J. COVID-19: European countries suspend use of Oxford-AstraZeneca vaccine after reports of blood clots. BMJ. 2021; 11, 372:n699. doi:10.1136/bmj.n699.

[41] Meredith S. More countries suspend AstraZeneca vaccinations over blood clot fears: What we know so far. CNBC. https://www.cnbc.com/2021/03/12/astrazeneca-covid-vaccine-suspended-in-some-countriesover-blood-clot-fears.html. Published March 12, 2021. Accessed June 8, 2021.

[42] PINHO AC. AstraZeneca's COVID-19 vaccine: EMA finds possible link to very rare cases of unusual blood clots with low platelets. European Medicines Agency. https://www.ema.europa.eu/en/news/astrazenecas-covid-19-vaccine-ema-finds-possible-linkvery-rare-cases-unusual-blood-clots-low-blood. Published April 8, 2021. Accessed June 8, 2021.

[43] The Gamaleya Center statement on increasing the interval between first and second Sputnik V vaccine shots. Sputnik V - the first registered vaccine against COVID-19. Official website vaccine against coronavirus Sputnik V. https://sputnikvaccine.com/ newsroom/pressreleases/the-gamaleya-center-statement-on-increasing-the-interval-between-first-and-second-sputnik-v-vaccine- $/ \#: \sim$ :text $=\% 22$ Regarding $\% 20$ the $\% 20$ Sputnik $\% 20 \mathrm{~V} \% 20$ vaccine,days $\% 20$ up $\% 20$ to $\% 20$ three\%20months. Accessed June 8, 2021.

[44] Sputnik V demonstrates $97.6 \%$ efficacy according to analysis of data from 3.8 million vaccinated persons in Russia making it the most efficient COVID-19 vaccine in the world. Sputnik V - the first registered vaccine against COVID-19. Official website vaccine against coronavirus Sputnik V. https://sputnikvaccine.com/newsroom/pressreleases/sputnik-vdemonstrates-97-6-efficacy-according-to-the-analysisof-data-of-3-8-million-vaccinated-per/. Accessed June 8, 2021.

[45] Tan BK, Mainbourg S, Friggeri A, et al. Arterial and venous thromboembolism in COVID-19: a study-level meta-analysis [published online ahead of print, $2021 \mathrm{Feb}$ 23]. Thorax. 2021; thoraxjnl-2020-215383. doi:10.1136/ thoraxjnl-2020-215383.
[46] Variants. COVID Reference. https://covidreference.com/variants. Accessed June 8, 2021.

[47] Gamaleya: Sputnik V. COVID19 Vaccine Tracker. https://covid19.trackvaccines.org/vaccines/12/. Accessed June 8, 2021.

[48] Vector vaccines AstraZeneca and Johnson \& Johnson have been given a conditional marketing authorisation by the EMA. What is their efficacy? Ge. https:// www.ge-bu.nl/en/article/vaccines-for-covid-19-vector-vaccines?full. Published April 30, 2021. Accessed June 8, 2021.

[49] The Gamaleya Center statement. Sputnik V - the first registered vaccine against COVID-19. Official website vaccine against coronavirus Sputnik V. https:// sputnikvaccine.com/newsroom/pressreleases/the-gamaleya-center-statement/. Accessed June 8, 2021.

[50] Single dose vaccine, Sputnik Light, authorized for use in Russia. Sputnik V - the first registered vaccine against COVID-19. Official website vaccine against coronavirus Sputnik V. https://sputnikvaccine.com/news$\mathrm{room} /$ pressreleases/single-dose-vaccine-sputnik-lightauthorized-for-use-in-russia/. Accessed June 8, 2021.

[51] Angola approves Russia's Sputnik Light one-dose vaccine. Reuters. https://www.reuters.com/article/ health-coronavirus-russia-angola-idUSR4N2L501M. Published May 12, 2021. Accessed June 8, 2021.

[52] McGregor G. It's not just Johnson \& Johnson: China has a single-dose COVID-19 vaccine that's $65 \%$ effective. Fortune. https://fortune.com/2021/02/09/china-covid-vaccine-single-dose-cansino-johnson-johnson/. Published February 24, 2021. Accessed June 9, 2021.

[53] Bloomberg.com. https://www.bloomberg.com/ news / articles / 2021-02-08/ pakistan-says-cansino-s-covid-vaccine-shows-65-7-efficacy?sref=XGjS8839. Accessed June 9, 2021.

[54] Reuters. CanSinoBIO says COVID-19 shot may be less effective over time, booster shot promising. Coronavirus. https://www.ctvnews.ca/health/coronavirus/cansinobio-says-covid-19-shot-may-be-less-effective-over-time-booster-shot-promising-1.5370940. Published April 1, 2021. Accessed June 9, 2021.

[55] CanSino: Ad5-nCoV. COVID19 Vaccine Tracker. https://covid19.trackvaccines.org/vaccines/2/. Accessed June 9, 2021.

[56] Chung YH, Beiss V, Fiering SN, Steinmetz NF. COVID-19 Vaccine Frontrunners and Their Nanotechnology Design. ACS Nano. 2020; 14 (10), 12522-37.

[57] Zhu FC, Li YH, Guan XH, et al. Safety, tolerability, and immunogenicity of a recombinant adenovirus type- 5 vectored COVID-19 vaccine: a dose-escalation, open-label, non-randomised, first-in-human trial. Lancet. 2020; 395 (10240), 1845-54.

[58] SARS-CoV-2 Variant Classifications and Definitions. Centers for Disease Control and Prevention. https://www.cdc.gov/coronavirus/2019-ncov/variants/variant-info.html. Accessed July 9, 2021. 
[59] Vasireddy D, Vanaparthy R, Mohan G, Malayala SV, Atluri P. Review of COVID-19 Variants and COVID-19 Vaccine Efficacy: What the Clinician Should Know? J Clin Med Res. 2021; 13 (6), 317-25.

[60] Pena L. As Delta variant turns into dominant strain in California, scientists detect 'Delta plus'. ABC7 San Francisco. https://abc7news.com/delta-variant-symptoms-of-virus-california-bay-area/10863428/. Published July 6, 2021. Accessed July 9, 2021.

[61] Coronavirus (COVID-19) Vaccinations - Statistics and Research. Our World in Data. https:/ / ourworldindata.org/covid-vaccinations. Accessed July 9, 2021.

[62] CDC COVID Data Tracker. Centers for Disease Control and Prevention. https://covid.cdc.gov/covid-data-tracker/\#vaccinations. Accessed July 7, 2021.
[63] World's first COVID-19 vaccine alternating dose study launches in UK. NIHR. https:/ / www.nihr.ac.uk/ news / worlds-first-covid-19-vaccine-alternating-dosestudy-launches-in-uk/26773. Accessed June 9, 2021.

[64] Callaway E. Mix-and-match COVID vaccines trigger potent immune response. Nature. 2021; 593 (7860), 491.

[65] He Q, Mao Q, An C, et al. Heterologous primeboost: breaking the protective immune response bottleneck of COVID-19 vaccine candidates. Emerg Microbes Infect. 2021; 10 (1), 629-37.

[66] Draft landscape of COVID-19 candidate vaccines. World Health Organization. https://www.who.int/ publications / m/item/draft-landscape-of-covid-19candidate-vaccines. Accessed January 17, 2021. 